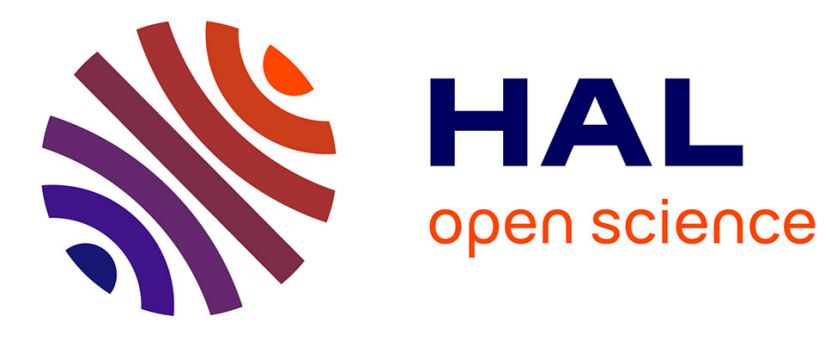

\title{
Phase Retrieval Techniques for Radar Ambiguity Problems
}

\author{
Philippe Jaming
}

\section{To cite this version:}

Philippe Jaming. Phase Retrieval Techniques for Radar Ambiguity Problems. Journal of Fourier Analysis and Applications, 1999, 5, pp.313-333. hal-00005818

\section{HAL Id: hal-00005818 https://hal.science/hal-00005818}

Submitted on 4 Jul 2005

HAL is a multi-disciplinary open access archive for the deposit and dissemination of scientific research documents, whether they are published or not. The documents may come from teaching and research institutions in France or abroad, or from public or private research centers.
L'archive ouverte pluridisciplinaire HAL, est destinée au dépôt et à la diffusion de documents scientifiques de niveau recherche, publiés ou non, émanant des établissements d'enseignement et de recherche français ou étrangers, des laboratoires publics ou privés. 


\title{
PHASE RETRIEVAL TECHNIQUES FOR RADAR AMBIGUITY PROBLEMS
}

\author{
PHILIPPE JAMING
}

\begin{abstract}
The radar ambiguity function plays a central role in the theory of radar signals. Its absolute value $(|A(u)|)$ measures the correlation between the signal $u$ emitted by the radar transmitter and its echo after reaching a moving target. It is important to know signals that give rise to ambiguity functions of given shapes. Therefore, it is also important to know to what extent $|A(u)|$ determines the signal. This problem is called the "radar ambiguity problem" by Bueckner 5 . Using methods developed for phase retrieval problems, we give here a partial answer for some classes of time limited (compactly supported) signals. In doing so, we also obtain results for Pauli's problem, in particular, we build functions that have infinitely many Pauli partners.
\end{abstract}

\section{INTRODUCTION}

In this paper three problems of reconstruction of lost phase are considered.

The first one arises in optics, in particular in the experimental use of diffraction to determine intrinsic structure. In this context, only the modulus of a Fourier transform can be measured after diffraction occurs. One is lead to the following problem.

Problem 1 (Phase Retrieval) Let $u_{1}, u_{2} \in L^{2}(\mathbb{R})$ be two compactly supported functions such that $\left|\mathcal{F} u_{1}(x)\right|=\left|\mathcal{F} u_{2}(x)\right|$ for all $x \in \mathbb{R}(\mathcal{F}$ being the Fourier transform $)$. Can one deduce $u_{2}$ from $u_{1}$ ?

Trivial solutions are $u_{2}(t)=c u_{1}(t+a)$ and $u_{2}(t)=c \overline{u_{1}(-t+a)}$ with $|c|=1$ and $a \in \mathbb{R}$. However, other solutions may arise. A fairly satisfying description of these solutions is given in Rosenblatt [18] and Walter [23]. For functions $u_{1}$ satisfying certain properties, there are only trivial solutions, but in general, the set of solutions is more complex.

One may ask if further conditions could imply uniqueness of the solutions. Such a question arises for instance in quantum mechanics. Pauli in his article on wave mechanics in the Handbuch der Physik [16] asked :

Problem 2 (Pauli Uniqueness) Is it possible, for a single particle moving in one dimension, to determine the wave function $\psi(q)$ from $|\psi(q)|$ and $|\mathcal{F} \psi(p)|$ ?

It seemed reasonable that this should be possible as $\psi(q)=|\psi(q)| e^{i \alpha(q)}$, thus only $\alpha(q)$ has to be found and one may think that the missing information is contained in $|\mathcal{F} \psi(q)|$. However this is not the case as shown by Bargman (cf. [17]) and later by Corbett and Hurst [7] and Vogt [22]. We will give new examples, and show in particular that a function may have an infinity of Pauli partners.

Another problem of the same kind occurs in the theory of radar signals and is known as the (Narrow Band) Radar Ambiguity Problem. The radar ambiguity function is the Fourier transform of the Wigner function : for $u \in L^{2}(\mathbb{R})$,

$$
A(u)(x, y)=\int_{\mathbb{R}} u\left(t+\frac{x}{2}\right) \overline{u\left(t-\frac{x}{2}\right)} e^{2 i \pi y t} d t .
$$

Here the only quantity that is actually measured by a radar is $|A(u)(x, y)|$ for $x, y \in \mathbb{R}$. Thus the following question arises :

Problem 3 (Radar Ambiguity) Let $u \in L^{2}(\mathbb{R})$, find all $v \in L^{2}(\mathbb{R})$ such that for all $x, y \in \mathbb{R}$,

$$
|A(v)(x, y)|=|A(u)(x, y)| .
$$

1991 Mathematics Subject Classification. 42B10, 81S30, 94A12.

Key words and phrases. radar ambiguity problem, Pauli partners, phase retrieval, Paley Wiener theorem. 
Trivial solutions are $v(t)=c e^{i \omega t} u(t+a)$ and $v(t)=c e^{i \omega t} u(-t-a)$ with $|c|=1, \omega, a \in \mathbb{R}$. The question arises whether they are the only solutions. Using algebraic methods, Bueckner [5] and de Buda [8] considered the case when $u$ is of the form $P(x) \exp \left(-\frac{x^{2}}{2}\right)$ with $P$ a polynomial. They proved that, in this case, almost every solution of the ambiguity problem is trivial ; de Buda also gave examples of functions for which there are non-trivial solutions of the ambiguity problem. We shall use here the methods developed for the phase retrieval problem to find all solutions to a restricted ambiguity problem. In doing so, we obtain new examples of functions for which there are only trivial solutions. We also throw new light on de Buda's counterexamples and show that they correspond to a "spectrum ambiguity".

Before going on, we introduce the following unitary operators $L^{2}(\mathbb{R}) \mapsto L^{2}(\mathbb{R})$.

(1) $\mathcal{F}$ is the Fourier transform,

(2) for $a \in \mathbb{R}, S(a) u(t)=u(t-a)$ - time shift -

(3) for $\omega \in \mathbb{R}, M(\omega) u(t)=e^{i \omega t} u(t)$ - frequency shift -

(4) for $\tau \in \mathbb{R} \backslash\{0\}, D_{\tau} u(t)=\sqrt{|\tau|} u(\tau t)$ - scaling operator -

(5) for $\lambda \in \mathbb{R}, L(\lambda) u(t)=e^{i \lambda \ln |t|} u(t)$.

(6) $Z u(t)=u(-t)$.

We will note $\langle u, v\rangle$ for the usual scalar product on $L^{2}(\mathbb{R})$ and $c$ will denote a complex number of modulus 1 . We find it convenient to note $C u(t)=\overline{u(t)}$. If $A \subset \mathbb{R}$ is a measurable set, we write $\chi_{A}$ for the caracteristic function of $A$ and $|A|$ for its Lebesgue measure.

Finally, we will also study the wide band ambiguity problem. The wide band ambiguity function is given by the formula

$$
W A(u)(\tau, a)=\sqrt{|\tau|} \int_{\mathbb{R}} u(t) \overline{u(\tau(t-a))} d t .
$$

We will study the equation $|W A(u)(\tau, a)|=|W A(v)(\tau, a)|$ for every $\tau>0, a \in \mathbb{R}$.

In this case, "trivial" solutions are of the form

(1) $v=c u$.

(2) $v=C Z u$.

(3) $v=\mathcal{F}^{-1} L(\lambda) \mathcal{F} u\left(\right.$ i.e. $\left.\mathcal{F} v(t)=e^{i \lambda \ln |t|} \mathcal{F} u(t)\right)$.

Using again the same tools, we show that these may be the only ones under appropriate conditions, and also provide an easy method to build counter-examples to the general problem (a "support ambiguity"). These results are new to our knowledge, except for the fact that the trivial solutions have been found by Altes [1] (however, he did not show that these may be the only ones).

The article is organised as follows. Section 2 is devoted to the phase retrieval problem and is a review of results by Rosenblatt [18] and Walter [23. In order to remain as self contained as possible we will recall the essential tools in the theory, i.e. the Paley-Wiener theorem and the Hadamard factorisation theorem, which will be used again in section 3. We will conclude this section with Pauli's problem and results by Corbett and Hurst [7], [6] and Vogt [22], and show how to build functions that have infinitely many Pauli partners. We will also list some connected questions which have probably not attracted the interest they deserve.

The last section is devoted to the radar ambiguity problems. After a quick review of various properties, we will present some results which are not, to our knowledge, available in the literature.

Before going on, we would like to attract the reader's attention to the monograph by Hurt [11] and to the survey article 15] devoted to the subject of phase retrieval.

We shall usually refrain from saying that equations are true almost everywhere.

\section{The Phase Retrieval Problem}

Recall that we want to determine, for a given compactly supported function $u \in L^{2}(\mathbb{R})$, every compactly supported function $v \in L^{2}(\mathbb{R})$ such that for every $x \in \mathbb{R},|\mathcal{F} u(x)|=|\mathcal{F} v(x)|$.

For this, the Paley Wiener theorem (see 114 theorem VI 7.4) provides us with important information about the form of $\mathcal{F} u$ when $u$ is compactly supported: 
Theorem (Paley Wiener) Let $F$ be an entire function and $a>0$.

The following conditions are equivalent :

(1) $\left.F\right|_{\mathbb{R}} \in L^{2}(\mathbb{R})$ and $|F(z)|=o\left(e^{a|z|}\right)$.

(2) There exists a function $f \in L^{2}(\mathbb{R})$ with $f(\xi)=0$ for $|\xi|>a$ such that

$$
F(z)=\frac{1}{2 \pi} \int_{\mathbb{R}} f(\xi) e^{i \xi z} d \xi .
$$

It follows that $\mathcal{F} u$ can be expanded into an entire function of exponential type (i.e. $|\mathcal{F} u(z)| \leq e^{B|z|}$ for $z \in \mathbb{C}$ ). We denote also by $\mathcal{F} u$ that expansion. By the Hadamard factorisation theorem (21] theorem 8.24) $\mathcal{F} u$ can be uniquely written in the form

$$
e^{\alpha_{0}+\alpha_{1} z} z^{k} \prod_{k=1}^{\infty}\left(1-\frac{z}{z_{k}}\right) e^{z / z_{k}}
$$

where the $z_{k}$ 's are the zeroes of $\mathcal{F} u$ in $\mathbb{C}$. Moreover, if we order the zeroes of $\mathcal{F} u$ so that $\left|z_{1}\right| \leq \ldots \leq$ $\left|z_{k}\right| \leq\left|z_{k+1}\right| \leq \ldots$, and if we write $z_{k}=r_{k}\left(\cos \theta_{k}+i \sin \theta_{k}\right)$, then

$$
\sum_{k \geq 1} \frac{1}{r_{k}^{1+\varepsilon}} \quad \text { converges for every } \varepsilon>0 .
$$

In particular, the infinite product $\prod_{k=1}^{\infty}\left(1-\frac{z}{z_{k}}\right) e^{z / z_{k}}$ is convergent.

It is important in what follows to note that for $z$ real,

$$
\left|\left(1-\frac{z}{z_{k}}\right) e^{z / z_{k}}\right|=\left|\left(1-\frac{z}{\overline{z_{k}}}\right) e^{z / \overline{z_{k}}}\right|
$$

Now as $v$ is also compactly supported, $\mathcal{F} v$ is also an entire function of order 1 , by the Hadamard factorisation theorem, it is (almost) characterised by its zeroes and admits a factorisation of the form

$$
e^{\beta_{0}+\beta_{1} z} z^{k} \prod_{k=1}^{\infty}\left(1-\frac{z}{\zeta_{k}}\right) e^{z / \zeta_{k}}
$$

It can then be shown that

Theorem 1 (Walter) Let $u \in L^{2}(\mathbb{R})$ be a compactly supported function. Let $\left\{z_{k}\right\}_{k \geq 1}$ be the zeroes of $\mathcal{F} u$ (counted with multiplicity, and ordered so that $\left|z_{k}\right|$ is an increasing sequence) and write

$$
\mathcal{F} u(z)=e^{\alpha_{0}+\alpha_{1} z} z^{k} \prod_{k=1}^{\infty}\left(1-\frac{z}{z_{k}}\right) e^{z / z_{k}} .
$$

Let $v \in L^{2}(\mathbb{R})$ be a compactly supported function, then $|\mathcal{F} u(x)|=|\mathcal{F} v(x)|$ for all $x \in \mathbb{R}$ if and only if there exists $c \in \mathbb{C}$ with $|c|=1, a \in \mathbb{R}$ and a choice $\zeta_{k} \in\left\{z_{k}, \overline{z_{k}}\right\}, k=1,2,3, \ldots$, such that for all $z \in \mathbb{C}$,

$$
\mathcal{F} v(z)=c e^{i a z} e^{\alpha_{0}+\alpha_{1} z} z^{k} \prod_{k=1}^{\infty}\left(1-\frac{z}{\zeta_{k}}\right) e^{z / \zeta_{k}} .
$$

Remark : Note that the coefficients $c$ and $a$ in the factorisation of $\mathcal{F} v$ in the above theorem are related to the trivial solution $v(t)=c u(t-a)$. Every choice of $\zeta_{k} \in\left\{z_{k}, \overline{z_{k}}\right\}$ gives rise to a non-trivial ambiguity, excepted when $\zeta_{k}=\overline{z_{k}}$ for every $k$ which corresponds to the trivial solution $v(t)=\overline{c u(-t+a)}$.

The possible replacement of $z_{k}$ by $\overline{z_{k}}$ is called "zero-flipping" by Walter.

If all the $z_{k}$ 's are real (i.e. $z_{k}=\overline{z_{k}}$ ) then only trivial solutions can occur. On the other hand, if at least two zeroes are non-real, there are non-trivial solutions. 
Note also that the zero-flipping corresponds to a factorisation $\mathcal{F} u(z)=f(z) g(z)$ and $\mathcal{F} v(z)=$ $f(z) \overline{g(\bar{z})}$. In higher dimension an entire function can be irreducible and the above factorisation may be trivial i.e. zero-flipping may be impossible, see [19], [4] for details.

Example 1: Let $H$ be a fixed function with support in $\left[-\frac{1}{2}, \frac{1}{2}\right]$. Let

$$
u(t)=\sum_{j=0}^{N} a_{j} H(t-j) \text { and } v(t)=\sum_{j=0}^{N} b_{j} H(t-j) .
$$

Then

$$
\begin{aligned}
& \mathcal{F} u(t)=P\left(e^{2 i \pi t}\right) \mathcal{F} H \text { with } P(X)=\sum_{j=0}^{N} a_{j} X^{j}, \text { and } \\
& \mathcal{F} v(t)=Q\left(e^{2 i \pi t}\right) \mathcal{F} H \text { with } Q(X)=\sum_{j=0}^{N} b_{j} X^{j} .
\end{aligned}
$$

To have $|\mathcal{F} u|=|\mathcal{F} v|$ it is enough that $|P|=|Q|$ on the unit circle.

But $|P|=|Q|$ on the unit circle if and only if $|P(1)|=|Q(1)|$ and,

$$
\left\{\alpha_{j}, \frac{1}{\overline{\alpha_{j}}}: \alpha_{j} \text { zero of } P\right\}=\left\{\alpha_{j}, \frac{1}{\overline{\alpha_{j}}}: \alpha_{j} \text { zero of } Q\right\}
$$

For instance, if we take

$$
\begin{aligned}
& P(X)=\left(\alpha X^{N}+\alpha^{\prime}\right)\left(\beta X+\beta^{\prime}\right) \\
& Q(X)=\left(\bar{\alpha}^{\prime} X^{N}+\bar{\alpha}\right)\left(\beta X+\beta^{\prime}\right)
\end{aligned}
$$

$\left(|\alpha| \neq\left|\alpha^{\prime}\right|,|\beta| \neq\left|\beta^{\prime}\right|\right)$ then $P$ and $Q$ are two different polynomials with $|P|=|Q|$, and $v$ is a non-trivial solution of the phase retrieval problem for $u$.

One might think that if we impose further conditions on the functions $u$ and $v$ such as regularity, or positivity, then $|\mathcal{F} u|=|\mathcal{F} v|$ could imply $u=c v$. However, the preceding example with $H$ a $\mathcal{C}^{\infty}$ function and $\alpha, \alpha^{\prime}, \beta, \beta^{\prime}>0$ shows that this is not the case.

The following example shows that if, in addition, we require that the support of $u$ be connected, we still do not force uniqueness.

Example 2: Let $f(x)=\exp \frac{1}{1-x^{2}}$ if $|x|<1$ and $f(x)=0$ if $|x| \geq 1$. Let $u=-f-f^{\prime}-f^{\prime \prime}$ and $v=-f+f^{\prime}-f^{\prime \prime}$. Then $u$ and $v$ are non-negative, with connected support and $\mathcal{C}^{\infty}$. However, $\mathcal{F} u=\left(4 \pi^{2} x^{2}-2 i \pi x-1\right) \mathcal{F} f$ and $\mathcal{F} v=\left(4 \pi^{2} x^{2}+2 i \pi x-1\right) \mathcal{F} f$, thus $|\mathcal{F} u|=|\mathcal{F} v|$.

Let us now consider the Pauli problem. Here again additional information is given. We know that $|\mathcal{F} u|=|\mathcal{F} v|$ and $|u|=|v|$. Hence the only trivial solutions are $v=c u$ with $|c|=1$. The previous approach will be difficult to use as zero-flipping on $\mathcal{F} u$ corresponds to a convolution of $u$ with a distribution, and it is far from obvious how to check that $|v|=|u|$.

We call $u$ Pauli unique if $|\mathcal{F} u|=|\mathcal{F} v|$ and $|u|=|v|$ implies $v=c u$. We say that $u$ and $v$ are Pauli partners if $|\mathcal{F} u|=|\mathcal{F} v|$ and $|u|=|v|$.

Reisenbach [17] published the examples of Pauli partners found by Bargman, but only in 1978 Vogt [22] and Corbett and Hurst [7], [6] first started a systematic study of the subject and showed that there are infinitely many Pauli unique functions as well as infinitely many Pauli partners. To exhibit Pauli partners, they used a method based on the relation $C \mathcal{F}=\mathcal{F} C Z$.

To be specific, let $u$ be a function such that $\overline{u(-t)}=u(t) w(t)$ with $|w(t)|=1$ and $w$ is not a constant on $\{t: u(t) \neq 0\}$ (that is, $|C Z u|=|u|$ ). Let $v=\mathcal{F}^{-1} C \mathcal{F} u=C Z u$. Then $|u|=|v|$, $|\mathcal{F} v|=|C \mathcal{F} u|=|\mathcal{F} u|$. Thus $u$ and $v$ are Pauli partners. Note also that $v$ is a trivial solution for the phase retrieval problem for $u$ !

Furthermore, if we take $|\alpha|=\left|\alpha^{\prime}\right|$ in example 1, then we obtain Pauli partners that are non trivial solutions of the phase retrieval problem. We prove more. 
Theorem 2 There exists $u \in L^{2}(\mathbb{R})$ which has a non-denumerable infinity of Pauli partners that are not trivial solutions of the phase retrieval problem.

Proof. Let $\left(a_{n}\right)$ be a sequence of compex numbers such that $\sum\left|a_{n}\right|^{2}$ converges and consider the Riesz product $F(x)=\prod_{n=0}^{\infty}\left(1+i a_{n} \sin 3^{n} x\right)$. Write $F(x)=\sum_{k=-\infty}^{\infty} b_{k} e^{i k x}$. Let $H \in L^{2}(\mathbb{R})$ be supported in $[0,1]$, and take

$$
u(t)=\sum_{k=-\infty}^{\infty} b_{k} H(t-k)
$$

Let $\varepsilon \in\{-1,1\}^{\mathbb{N}}$ and $F^{(\varepsilon)}(x)=\prod_{n=0}^{\infty}\left(1+i a_{n} \varepsilon_{n} \sin 3^{n} x\right)$. Write $F^{(\varepsilon)}(x)=\sum_{k=-\infty}^{\infty} b_{k}^{(\varepsilon)} e^{i k x}$ Then $v^{(\varepsilon)}=\sum_{k=-\infty}^{\infty} b_{k}^{(\varepsilon)} H(t-k)$ is a Pauli partner for $u$.

For properties of Riesz products, see [14].

Remark 1: In the above example, if we take $H$ a $\mathcal{C}^{\infty}$ function and $\left(a_{k}\right)$ a fastly decreasing sequence, one can even obtain $u$ in the Schwartz class.

Remark 2: This theorem has already be found in a less precise form by Ismagilov [12] (a fact we have not been aware of while writing the first version of this article). It answers a question asked by Friedman [9].

One may then ask if further conditions could imply unicity. More precisely, the following questions arise.

Problem 4 Find unitary operators $U_{1}, \ldots, U_{n}: L^{2}(\mathbb{R}) \mapsto L^{2}(\mathbb{R})$ (not necessarily commuting) such that if $u, v \in L^{2}(\mathbb{R})$ satisfy $|u|=|v|,|\mathcal{F} u|=|\mathcal{F} v|$ and $\left|U_{i} u\right|=\left|U_{i} v\right|(i=1, \ldots n)$, then $v=c u$.

Problem 4bis Given $u \in L^{2}(\mathbb{R})$, find unitary operators $U_{1}, \ldots, U_{n}: L^{2}(\mathbb{R}) \mapsto L^{2}(\mathbb{R})$ (not necessarily commuting) such that if $v \in L^{2}(\mathbb{R})$ satisfies $|u|=|v|,|\mathcal{F} u|=|\mathcal{F} v|$ and $\left|U_{i} u\right|=\left|U_{i} v\right|(i=1, \ldots n)$, then $v=c u$.

If such operators were found, it would also be important to give them a physical meaning.

Note that an operator that commutes with $C$ as well as the identity operator and the Fourier transform will not work (22]).

\section{The Radar Ambiguity Problem}

3.1. The problem. As radar computations are not familiar to the general mathematical community, we begin with a brief simplified version of the way ambiguity functions arise in radar theory. In doing so, we essentially follow Wilcox [24] and Auslander and Tolimieri [3] for the narrow band ambiguity function and Auslander and Gertner [2] for the wide band case.

Let $X$ be an object or target and assume the radar is at the origin. Let $r(t)$ denote the distance from $X$ to the radar and let $v(t)$ denote the radial velocity of $X$ at time $t$. The problem is to transmit an electromagnetic wave or pulse $s(t),-T<t<T$, and from the echo $e(t)$ obtained after reflection on the target, determine the quantities $r(0)$ and $v(0)$.

We will now briefly explain how information is extracted from $e(t)$. The first step is to pass from the pulse to a complex valued function called the waveform of the pulse. As $s(t)$ is real valued, we have $\mathcal{F} s(-t)=\overline{\mathcal{F} s(t)}$, and so $s(t)$ is completely determined by its positive spectrum. Define the operator $\psi: L^{2}(\mathbb{R}) \mapsto L^{2}(\mathbb{R})$ by

$$
s \mapsto \psi s(x)=\int_{0}^{\infty} \mathcal{F} s(t) e^{2 i \pi x t} d t .
$$

Then $\psi s(t)=\frac{s(t)+i \sigma(t)}{2}$ where $\sigma$ is the Hilbert transform of $s$. Explicitly

$$
\sigma(x)=\frac{1}{\pi} \lim _{\varepsilon \rightarrow 0} \int_{|x-t|>\varepsilon} \frac{s(t)}{x-t} d t .
$$

We have $\|\psi s\|_{L^{2}}^{2}=\frac{1}{2}\|s\|_{L^{2}}^{2}$. It is customary to call $\|f\|_{L^{2}}^{2}$ the energy of the signal $s$.

Assume now that $s$ satisfies the following properties, 
(1) $\|\psi s\|=1$,

(2) $\int_{-\infty}^{\infty}|t \psi s(t)|^{2} d t<\infty$,

(3) and $\int_{-\infty}^{\infty}|t \mathcal{F} \psi s(t)|^{2} d t<\infty$.

Let

$$
t_{0}=\int_{-\infty}^{\infty} t|\psi s(t)|^{2} d t, \quad f_{0}=\int_{-\infty}^{\infty} t|\mathcal{F} \psi s(t)|^{2} d t
$$

$t_{0}$ is called the epoch and $f_{0}$ the carrier frequency.

We will further assume that $s$ is narrow banded, i.e. that the spectrum of $s$ is small compared to the doppler shift due to the radial movement of the target. This validates the narrow band approximation, i.e. the replacement of the Doppler scaling of the spectrum by a shift.

Definition The waveform $u_{s}(t)$ of the pulse $s(t)$ is defined by

$$
u_{s}(t)=\psi s\left(t+t_{0}\right) e^{-2 i \pi f_{0}\left(t+t_{0}\right)}
$$

It follows that $s(t)=\Re\{\psi s(t)\}=\Re\left\{u_{s}\left(t-t_{0}\right) e^{2 i \pi f_{0} t}\right\}$ and that $\left\|u_{s}\right\|_{L^{2}}^{2}=1$. The waveform $u_{s}(t)$ is "slowly varying" in the sense that its spectrum is centered around the 0 frequency.

Assume the following physical conditions are satisfied :

(1) There is only one target.

(2) The radar cross section of the target is independent of frequency.

(3) The target is in the far field of the radar.

(4) Multiple reflecting waves are negligible.

(5) The function $r(t)$ is approximately linear for $-T<t<T$.

(6) The velocity of the target is small compared to the speed of electromagnetic propagation.

Then it is generally accepted that the echo is given by $e(t)=\Re\{\psi e(t)\}$, with

where

$$
\psi e(t)=e^{-2 \pi i f_{0} x_{0}} u_{s}\left(t-t_{0}-x_{0}\right) e^{2 i \pi\left(f_{0}-y_{0}\right) t}
$$

$$
\begin{aligned}
& x_{0}=\frac{2}{c} r(0) \text { is called the time delay of the echo } \\
& y_{0}=\frac{2 f_{0}}{c} v(0) \text { is the Doppler or frequency shift of the echo }
\end{aligned}
$$

with $c$ the velocity of light. In particular, $x_{0}, y_{0}$ completely determine $r(0)$ and $v(0)$.

One then estimates $x_{0}, y_{0}$ by the following method originally suggested by Woodward 25]. Consider

and form

$$
\psi_{x y}(t)=e^{-2 i \pi f_{0} x} u_{s}\left(t-t_{0}-x\right) e^{-2 i \pi y t} e^{2 i \pi f_{0} t}
$$

$$
I(x, y)=\left|\int_{-\infty}^{+\infty} \psi e(t) \overline{\psi_{x y}(t)} d t\right|^{2} .
$$

As $\left\|u_{s}\right\|^{2}=1, I\left(x_{0}, y_{0}\right)=1$ and $I(x, y) \leq 1$ for all $x, y$. Thus if we regard $I(x, y)$ as light intensity on a screen, the brightest point should be $\left(x_{0}, y_{0}\right)$ enabling us to determine the range and velocity of the target. It is crucial for us to observe that

where

$$
I(x, y)=\left|A\left(u_{s}\right)\left(x_{0}-x, y_{0}-y\right)\right|^{2}
$$

$$
A(u)(x, y)=\int_{\mathbb{R}} u\left(t+\frac{x}{2}\right) \overline{u\left(t-\frac{x}{2}\right)} e^{2 i \pi y t} d t
$$


One would like to make sure, in this context, that the observed quantity, which arises from $u_{s}$, corresponds to the given radar signal or, depending on the aims of the user, to find $v_{s}$ such that $\left|A\left(v_{s}\right)\right|=\left|A\left(u_{s}\right)\right|$. Following Bueckner [5], we formulate :

Problem (Radar Ambiguity) Given $u \in L^{2}(\mathbb{R})$, what is the set of all functions $v \in L^{2}(\mathbb{R})$ such that

$$
|A(v)(x, y)|=|A(u)(x, y)|
$$

for all $x, y \in \mathbb{R}$ ?

If $v$ is such a function, we say that $v$ is an ambiguity partner of $u$.

Unfortunately, in many cases such as signals arising in seismology, oceanography and sonar, the narrow-band approximation is not valid. Thus, when the target is moving, there is no uniform Doppler shift across the entire spectra. In [20], a signal model independent of bandwidth and central frequency was proposed. Following Auslander and Gertner [2], we will now describe this model. This time

$$
\psi e(t)=A \psi s(a t-\tau)
$$

where

$$
\begin{aligned}
& A--- \text { The energetic parameter of the signal } \\
& a=\frac{c-v(0)}{c+v(0)}--- \text { The Doppler stretch }- \text { compress factor } \\
& \tau=\frac{2 r(0)}{c+v(0)}--- \text { The signal delay at time } t=0 .
\end{aligned}
$$

(Note that $a>0$ ). We shall ignore signal attenuation and we will again normalise the energy to 1 . We obtain

$$
1=\int_{-\infty}^{+\infty}|e(t)|^{2} d t=A^{2} \int_{-\infty}^{+\infty}|\psi s(a t-\tau)|^{2} d t=\frac{A^{2}}{a} \int_{-\infty}^{+\infty}|\psi s(t)|^{2} d t=\frac{A^{2}}{a} .
$$

Thus conservation of energy requires that $A=\sqrt{a}$, so the received signal can be represented as

with $\tau^{\prime}=\frac{2 r(0)}{c-v(0)}$.

$$
\psi e(t)=\sqrt{a} \psi s(a t-\tau)=\sqrt{a} \psi s\left(a\left(t-\tau^{\prime}\right)\right)
$$

The problem is to estimate the Doppler parameter $a$ and the delay $\tau$ (that determine $r(0)$ and $v(0)$ ). The maximum likelihood estimate of these parameters is obtained by finding the maximum of the cross correlation function

$$
\max _{a, \tau}\left|\sqrt{a} \int_{-\infty}^{+\infty} \psi s(t) \overline{\psi s(a(t-\tau))} d t\right|^{2} .
$$

This suggests that the definition of the wide band ambiguity function be

$$
W A(u)(a, \tau)=\sqrt{a} \int_{\mathbb{R}} u(t) \overline{u(a(t-\tau))} d t
$$

We also formulate the wide band analogue of the ambiguity problem.

Problem (Wide Band Radar Ambiguity) Given $u \in L^{2}(\mathbb{R})$, what is the set of all $v \in L^{2}(\mathbb{R})$ such that

$$
|W A(v)(x, y)|=|W A(u)(x, y)|
$$

for all $x>0, y \in \mathbb{R}$ ?

If $v$ is such a function, we will say that $v$ is a wide band ambiguity partner of $u$. 
For both ambiguity problems, the complete answer is unknown. However, for the narrow band case, it may easily be checked that possible choices of $u$ are :

(1) $v=c u$, where $c$ is a complex number of modulus 1 .

(2) $v=M(\omega) u, \omega \in \mathbb{R}$.

(3) $v=S(a) u, a \in \mathbb{R}$.

(4) $v=Z u$.

More cases may be obtained by combinations of such transformations. We will say that $v$ is Heisenberg related to $u$ if $v$ is obtained from $u$ by a sequence of transformations like 1, 2, 3, 4 .

In the wide band case, the situation is more complicated. First note that

$$
W A(u)(a, \tau)=<u, D_{a} S(\tau) u>
$$

With Parseval's identity and the commutation relations between $\mathcal{F}, D$ and $S$, we obtain

where

$$
\begin{aligned}
W A(u)(a, \tau) & =<\mathcal{F} u, \mathcal{F} D_{a} S(\tau) u>=<\mathcal{F} u, D_{1 / a} \mathcal{F} S(\tau) u> \\
& =<\mathcal{F} u, M\left(\frac{\tau}{a}\right) D_{1 / a} \mathcal{F} u> \\
& =\widetilde{W A}(\mathcal{F} u)\left(\frac{1}{a}, \frac{\tau}{a}\right)
\end{aligned}
$$

$$
\widetilde{W A}(u)(a, \tau)=\frac{1}{\sqrt{a}} \int_{\mathbb{R}} u(t) \overline{u(a t)} e^{2 i \pi t \tau} d t .
$$

Trivial solutions of the wide band ambiguity problem are then

(1) $v=c u$ with $|c|=1$.

(2) $v=C Z u$.

(3) $v=\mathcal{F}^{-1} L(\lambda) \mathcal{F} u\left(\right.$ i.e. $\left.\mathcal{F} v(t)=e^{i \lambda \ln |t|} \mathcal{F} u(t)\right)$, with $\lambda \in \mathbb{R}$

If $v$ is obtained from $u$ by a sequence of transformations of that type, we will say that $v$ is A-related to $u$.

We will not directly solve the wide band ambiguity problem. Instead, we rather solve the problem for $\widetilde{W A}$, i.e. we ask the following question :

Problem ( $\widetilde{W A}$ Ambiguity Problem) Given $u \in L^{2}(\mathbb{R})$, what is the set of all $v \in L^{2}(\mathbb{R})$ such that

for all $a>0, \tau \in \mathbb{R}$ ?

$$
|\widetilde{W A}(u)(a, \tau)|=|\widetilde{W A}(v)(a, \tau)|
$$

The trivial solutions of this problem are then

(1) $v=c u$ with $|c|=1$.

(2) $v=C u$.

(3) $v=L(\lambda) u$ with $\lambda \in \mathbb{R}$.

3.2. The Narrow Band Case. We will here deal with the narrow band case and detail the proofs before giving results in the wide band case. But first, we note that $u$ and $v$ may be ambiguity partners without $v$ being Heisenberg related to $u$.

For instance, De Buda [8] gave the following example. Let

$$
u(t)=\left(\frac{\sin t}{t}\right)^{n} \sin (2 \pi n t) \quad \text { and } \quad v(t)=\left(\frac{\sin t}{t}\right)^{n} \cos (2 \pi n t)
$$

(for $n \geq 1$ ). Then $u$ and $v$ are ambiguity partners even though $v$ is not Heisenberg related to $u$.

Nevertheless, for special classes of functions $u$ it is the case that every ambiguity partner of $u$ is Heisenberg related to $u$. For instance, Bueckner ([5]) and De Buda ([8]) proved that this is the case if $u$ is of the form $P(x) \exp \left(-\frac{x^{2}}{2}\right)$ with $P$ a polynomial. We conjecture that this result should extend to functions of the form $P(x) \exp \left(-\frac{x^{2}}{2}\right)$ with $P$ an entire function of order $\alpha<2$. 
We start with an easy way to obtain counter-examples (which includes De Buda's counterexample), and then provide some positive results.

3.2.1. Counter-examples. Let $u_{1}, u_{2}$ be two functions in $L^{2}(\mathbb{R})$, respectively supported in $A_{1}$ and $A_{2}$, and let $c_{1}, c_{2}$ be two complex numbers with $\left|c_{1}\right|=\left|c_{2}\right|=1$. We then have

$$
\begin{aligned}
A\left(c_{1} u_{1}+c_{2} u_{2}\right) & =\left|c_{1}\right|^{2} A\left(u_{1}\right)+\left|c_{2}\right|^{2} A\left(u_{2}\right)+c_{1} \overline{c_{2}} A\left(u_{1}, u_{2}\right)+\overline{c_{1}} c_{2} A\left(u_{2}, u_{1}\right) \\
& =A\left(u_{1}\right)+A\left(u_{2}\right)+c_{1} \overline{c_{2}} A\left(u_{1}, u_{2}\right)+\overline{c_{1}} c_{2} A\left(u_{2}, u_{1}\right)
\end{aligned}
$$

where $A\left(u_{1}, u_{2}\right)$ is the bilinear operator associated to $A(u)$. Thus, if we can choose the sets $A_{1}$ and $A_{2}$ so that $A\left(u_{1}, u_{2}\right), A\left(u_{2}, u_{1}\right)$ and $A\left(u_{1}\right)+A\left(u_{2}\right)$ have disjoint supports, we obtain

$$
\begin{aligned}
\left|A\left(c_{1} u_{1}+c_{2} u_{2}\right)\right| & =\left|A\left(u_{1}\right)+A\left(u_{2}\right)\right|+\left|A\left(u_{1}, u_{2}\right)\right|+\left|A\left(u_{2}, u_{1}\right)\right| \\
& =\left|A\left(u_{1}+u_{2}\right)\right|
\end{aligned}
$$

though $c_{1} u_{1}+c_{2} u_{2}$ need not be Heisenberg related to $u_{1}+u_{2}$.

Now note that the support of

$$
t \mapsto u_{1}\left(t+\frac{x}{2}\right) \overline{u_{2}\left(t-\frac{x}{2}\right)}
$$

is included in $\left(A_{1}-\frac{x}{2}\right) \cap\left(A_{2}+\frac{x}{2}\right)$; in particular, $u_{1}\left(t+\frac{x}{2}\right) \overline{u_{2}\left(t-\frac{x}{2}\right)}=0$ for every $t \in \mathbb{R}$ if $x \notin$ $A_{1}-A_{2}$, so that the support of $A\left(u_{1}, u_{2}\right)$ is included in the strip $\left(A_{1}-A_{2}\right) \times \mathbb{R}$. Similarly, the support of $A\left(u_{2}, u_{1}\right)$ is included in the strip $\left(A_{2}-A_{1}\right) \times \mathbb{R}$. Thus, $A\left(u_{1}, u_{2}\right)$ and $A\left(u_{2}, u_{1}\right)$ have disjoint support if $A_{1}-A_{2}$ and $A_{2}-A_{1}$ are disjoint.

In a similar way, we also need $A_{1}-A_{2}$ to be disjoint from $A_{1}-A_{1}$ and from $A_{2}-A_{2}$. The condition that $A_{2}-A_{1}$ is disjoint from $A_{1}-A_{1}$ and from $A_{2}-A_{2}$ then follows automatically.

Finally we notice that there is no reason to restrict ourself to two functions. We can then state the following :

Proposition 3 Let $\left\{A_{j}\right\}_{j \geq 1}$ be a disjoint family of subsets of $\mathbb{R}$, of positive measure, satisfying the following conditions

(1) the family $\left\{A_{i}-A_{j}\right\}_{i \neq j}$ is disjoint,

(2) for $i<j$ and for all $k, A_{j}-A_{i}$ is disjoint from $A_{k}-A_{k}$.

Let $\left(u_{i}\right)_{i \geq 1}$ be a family of functions such that $u_{i}$ is supported in $A_{i}$ and such that $\sum_{i \geq 1} u_{i} \in L^{2}(\mathbb{R})$.

Finally let $\left(c_{i}\right)_{i \geq 1}$ be a family of complex numbers of modulus 1 . Then $\sum_{i \geq 1} c_{i} u_{i}$ and $\sum_{i \geq 1} u_{i}$ are ambiguity partners but $\sum_{i \geq 1} c_{i} u_{i}$ is generally not Heisenberg related to $\sum_{i \geq 1} u_{i}$.

It is easy (see [3]) to see that $A(\mathcal{F} u)(x, y)=A(u)(-y, x)$ and we could state a similar proposition for the spectrum of $u$ instead of the support. In particular, De Buda's counter example is obtained with $A_{1}=[-2 n,-n], A_{2}=[n, 2 n](n \geq 1), \mathcal{F} u=\chi_{A_{1}}+\chi_{A_{2}}$ and $\mathcal{F} v=\chi_{A_{1}}-i \chi_{A_{2}} .{ }^{1}$

A slight modification of that method, inspired by example 1 , leads us to the following example which will be crucial to us.

Example $3:$ Let $H \in L^{2}(\mathbb{R})$ be supported in $\left[0, \frac{1}{2}\right]$ and let $\left(a_{j}\right)_{j=0, \ldots, n}$ be a sequence of complex numbers. Let $u(t)=\sum_{j=0}^{n} a_{j} H(t-j)$. Using the bilinearity of $A$ as before, it is easy to see that, for $x \in\left[j-\frac{1}{2}, j+\frac{1}{2}\right], j=0 \ldots, n$,

$$
\begin{aligned}
& \qquad A(u)(x, y)=e^{i \pi j y}\left(\sum_{k=j}^{n} a_{k} \overline{a_{k-j}} e^{2 i \pi k y}\right) A(H)(x-j, y), \\
& \text { while } A(u)(x, y)=0 \text { for }|x|>N+\frac{1}{2} \text {, and } A(u)(-x,-y)=\overline{A(u)(x, y)} \text {. }
\end{aligned}
$$

\footnotetext{
${ }^{1}$ Note added in proof : F.A. Grüenbaum [10] found the example $u_{1}=\chi_{[-1,1]}, u_{2}=\chi_{[4,5]}, u_{3}=\chi_{[-5,-4]}$.
} 
If $v(t)=\sum_{j=0}^{n} b_{j} H(t-j)$, then $u$ and $v$ are ambiguity partners if and only if for $j=0, \ldots, n$

$$
\left|\sum_{k=j}^{n} a_{k} \overline{a_{k-j}} e^{2 i \pi k y}\right|=\left|\sum_{k=j}^{n} b_{k} \overline{b_{k-j}} e^{2 i \pi k y}\right| .
$$

Let $N \geq 3$, and choose $u(t)=a_{0} H(t)+a_{1} H(t-1)+a_{N} H(t-N)+a_{N+1} H(t-N-1)$ and $v(t)=b_{0} H(t)+b_{1} H(t-1)+b_{N} H(t-N)+b_{N+1} H(t-N-1)$. Then, to have ambiguity partners, we need

(1) for $j=0,\left|a_{0}\right|^{2}+\left|a_{1}\right|^{2} X+\left|a_{N}\right|^{2} X^{N}+\left|a_{N+1}\right|^{2} X^{N+1}$ and $\left|b_{0}\right|^{2}+\left|b_{1}\right|^{2} X+\left|b_{N}\right|^{2} X^{N}+\left|b_{N+1}\right|^{2} X^{N+1}$ to have same modulus on the unit circle $\mathbb{T}$. Following example 1 , if we take $\alpha, \alpha^{\prime}, \beta, \beta^{\prime}>0$ and the $a_{i}$ 's and $b_{i}$ 's such that

$$
\left|b_{0}\right|^{2}=\alpha \beta^{\prime} \quad\left|b_{1}\right|^{2}=\alpha \beta \quad\left|b_{N}\right|^{2}=\alpha^{\prime} \beta^{\prime} \quad\left|b_{N+1}\right|^{2}=\alpha^{\prime} \beta
$$

then, for $-\frac{1}{2} \leq x<\frac{1}{2}, y \in \mathbb{R}$, we have $|A(u)(x, y)|=|A(v)(x, y)|$.

(2) for $j=1, a_{0} \bar{a}_{1}+a_{N} \bar{a}_{N+1} X^{N}$ and $b_{0} \bar{b}_{1}+b_{N} \bar{b}_{N+1} X^{N}$ to have the same modulus on $\mathbb{T}$. But, according to 6 6 , $\left|a_{N} \bar{a}_{N+1}\right|=\left|b_{0} \bar{b}_{1}\right|$, so that the two polynomials have the same modulus on $\mathbb{T}$ if

$$
\frac{a_{0} \bar{a}_{1}}{a_{N} \bar{a}_{N+1}}+X^{N} \quad \text { and } 1+\frac{b_{N} \bar{b}_{N+1}}{b_{0} \bar{b}_{1}} X^{N}
$$

have same modulus on $\mathbb{T}$, which is the case when

$$
\frac{b_{N} \bar{b}_{N+1}}{b_{0} \bar{b}_{1}}=\frac{\overline{a_{0} \bar{a}_{1}}}{a_{N} \bar{a}_{N+1}} .
$$

Note that by equation 647, these two complex numbers have the same modulus. Thus this reduces to the following condition on the phases :

$$
\begin{aligned}
& \arg b_{0}-\arg b_{1}-\arg b_{N}+\arg b_{N+1} \\
& \quad \equiv \arg a_{0}-\arg a_{1}-\arg a_{N}+\arg a_{N+1}
\end{aligned}
$$

(3) for $j=2, \ldots, N-2$, there is no extra condition.

(4) for $j=N-1,\left|a_{N} \bar{a}_{1} X^{N}\right|=\left|b_{N} \bar{b}_{1} X^{N}\right|$ on $\mathbb{T}$, which is a consequence of condition 66 .

(5) for $j=N,\left|a_{N} \bar{a}_{0}+a_{N+1} \bar{a}_{1} X\right|=\left|b_{N} \bar{b}_{0}+b_{N+1} \bar{b}_{1} X\right|$ on $\mathbb{T}$. As $\left|a_{N} \bar{a}_{0}\right|=\left|b_{N} \bar{b}_{0}\right|$, we need $\left|1+\frac{a_{N+1} \bar{a}_{1}}{a_{N} \bar{a}_{0}} X\right|=\left|1+\frac{b_{N+1} \bar{b}_{1}}{b_{N} \bar{b}_{0}} X\right|$, that is

$$
\frac{a_{N+1} \bar{a}_{1}}{a_{N} \bar{a}_{0}}=\frac{b_{N+1} \bar{b}_{1}}{b_{N} \bar{b}_{0}} .
$$

Again, condition 6f implies that these two complex numbers have the same modulus while condition 8 implies that they have the same argument.

(6) finally, for $j=N+1,\left|a_{N+1} \bar{a}_{0}\right|=\left|b_{N+1} \bar{b}_{0}\right|$, which follows from equations 647 .

It is important in what follows to note that, by the Paley Wiener theorem, $A(u)$ and $A(v)$ are entire functions in the $y$ variable but $A(u)$ does not have the same zeroes as $A(v)$ or $A(Z v)=\overline{A(v)}$.

Also note that we could modify the above example for $N=2$ and still obtain ambiguity partners (the only modification would be for $j=1$ ), but, in addition, the support of $A(u)$ would be connected. 
3.2.2. The Radar Ambiguity Problem for compactly supported functions. Let $u \in L^{2}(\mathbb{R})$ be a compactly supported function. We will now examine some conditions for the solutions $v$ of $|A(v)|=|A(u)|$ to be Heisenberg related to $u$. First we need to see that $v$ is also compactly supported.

Lemma 4 Let $u \in L^{2}(\mathbb{R})$ be a compactly supported function. Let $v \in L^{2}(\mathbb{R})$ satisfy $|A(v)(x, y)|=$ $|A(u)(x, y)|$ for all $x, y \in \mathbb{R}$. Then $v$ is also compactly supported.

Moreover if the support of $u$ is contained in an interval of length $2 a$ then the support of $v$ is also contained in an interval of length $2 a$.

Proof. Up to a time shift $S(\alpha)$, we may assume that $u$ is supported in $[-a, a]$, then, if $|x|>2 a$, we have, for all $t \in \mathbb{R}, u\left(t+\frac{x}{2}\right) \overline{u\left(t-\frac{x}{2}\right)}=0$ thus

$$
A(u)(x, y)=\int_{-\infty}^{\infty} u\left(t+\frac{x}{2}\right) \overline{u\left(t-\frac{x}{2}\right)} e^{i y t} d t=0
$$

for all $y \in \mathbb{R}$ and all $x$ with $|x|>2 a$.

But then, as $|A(v)(x, y)|=|A(u)(x, y)|$ for $x, y \in \mathbb{R}$, we get $A(v)(x, y)=0$ for all $y \in \mathbb{R}$ and all $x$ with $|x|>2 a$.

Now, noticing that $A(v)(x, y)$ is the Fourier transform of

we obtain

$$
v\left(t+\frac{x}{2}\right) \overline{v\left(t-\frac{x}{2}\right)}
$$

$$
v\left(t+\frac{x}{2}\right) \overline{v\left(t-\frac{x}{2}\right)}=0 \text { for all } t, \text { and all } x \text { with }|x|>2 a .
$$

After a change of variables, we deduce from this that $v\left(x_{1}\right) v\left(x_{2}\right)=0$ as soon as $\left|x_{1}-x_{2}\right|>2 a$. Therefore, the support of $v$ is contained in an interval of length $2 a$.

Remark : This lemma actually gives more information. Up to a translation, we may assume that the support of $u$ is contained in $[-a, a]$ and in no smaller interval. Then, by the lemma, the support of $v$ is contained in an interval of length $2 a$ that, up to a translation, we may assume to be $[-a, a]$. Assume that $v$ is actually supported in a smaller interval of length, say $2 b<2 a$; then we may exchange the roles of $u$ and $v$ to obtain that the support of $u$ is contained in an interval of length $2 b$, that is smaller interval than $[-a, a]$. This gives a contradiction.

We may now assume that supports of both $u$ and $v$ are contained in $[-a, a]$ and no smaller interval, in particular, $u$ and $v$ are compactly supported. Thus Paley Wiener theorem ensures us that $A(u)(x, y)$ and $A(v)(x, y)$ are both entire functions of exponential type in the $y$ variable. But $|A(u)(x, y)|=$ $|A(v)(x, y)|$ for all $x, y \in \mathbb{R}$, thus $A(u)(x, y) \overline{A(u)(x, y)}=A(v)(x, y) \overline{A(v)(x, y)}$ and then by analytic continuation we find that

$$
A(u)(x, y) \overline{A(u)(x, \bar{y})}=A(v)(x, y) \overline{A(v)(x, \bar{y})} \text { for all } x \in \mathbb{R}, y \in \mathbb{C} .
$$

On the other hand, due to the Hadamard factorisation theorem, an entire function $f(z)$ of exponential type is entirely determined by its zeros, up to a factor $\lambda e^{\mu z}$ with $\lambda, \mu \in \mathbb{C}$. Unfortunately 9 only tells us that, for fixed $x$, if $z$ is a zero of $A(u)(x,$.$) then either z$ or $\bar{z}$ is a zero of $A(v)(x,$.$) (zero flipping).$

Several cases occur, for instance, $A(u)$ may only have real zeros (e.g. if $\left.u=\chi_{[a, b]}\right)$, then $A(u)$ and $A(v)$ have the same zeroes.

There are some functions $u$ for which every ambiguity partner $v$ is such that either $A(u)$ and $A(v)$ have the same zeroes, or $A(u)$ and $A(Z v)$ have the same zeroes. We do not know if this can occur when $A(u)$ has non-real zeroes, and so we are unable to give a characterisation of the functions $u$ for which this is the case.

Finally, as in example $3, A(u)$ and $A(v)$ may have some common non-real zeroes and some conjugate zeroes.

In what follows, after eventually replacing $u$ by $Z u$ or by some $u_{f}$ if zero flipping occurs, we shall assume that $A(u)$ and $A(v)$ have the same zeroes. In other words we now consider the following restricted radar ambiguity problem : 
Problem (Restricted Radar Ambiguity) Given a compactly supported $u \in L^{2}(\mathbb{R})$, what is the set of ambiguity partners $v$ of $u$, such that for every $x \in \mathbb{R}, A(u)(x,$.$) and A(v)(x,$.$) have the same$ zeroes in the complex plane?

We will call such ambiguity partners restricted ambiguity partners.

Example 3 shows that :

Proposition 5 There exist compactly supported functions $u$ which have ambiguity partners that are not restricted ambiguity partners either of $u$ or of $Z u$.

By the Hadamard factorisation theorem, if $u$ and $v$ are restricted ambiguity partners, we have that, for each $x \in \mathbb{R}$, there exist two complex numbers $\lambda_{x}, \mu_{x}$ such that, for all $y \in \mathbb{C}, A(v)(x, y)=$ $\lambda_{x} e^{\mu_{x} y} A(u)(x, y)$, as these two ambiguity functions have same zeroes. Further, as $|A(u)(x, y)|=$ $|A(v)(x, y)|$, we see that $\left|\lambda_{x}\right|=1$ and $\mu_{x}$ is a purely imaginary complex number. We can, therefore, write

$$
A(v)(x, y)=e^{i \varphi(x)+i \psi(x) y} A(u)(x, y) \quad \text { for all } x, y \in \mathbb{R}
$$

where $\varphi, \psi: \mathbb{R} \mapsto \mathbb{R}$.

We first identify $\psi$. (10) implies that $A(v)(0, y)=A(u)(0, y)$ for every $y \in \mathbb{R}$. But $A(u)(0, y)=$ $\mathcal{F}\left[|u|^{2}\right](y)$ and $A(v)(0, y)=\mathcal{F}\left[|v|^{2}\right](y)$, thus $|u|^{2}=|v|^{2}$. In particular $u$ and $v$ have same support, so for every $x \in \mathbb{R}$,

$$
t \mapsto u\left(t+\frac{x}{2}\right) \overline{u\left(t-\frac{x}{2}\right)}, \text { and } t \mapsto v\left(t+\frac{x}{2}\right) \overline{v\left(t-\frac{x}{2}\right)}
$$

have same support. But, the Fourier transforms of these functions are $A(u)(x,$.$) and A(v)(x,$.$) thus,$ by the Paley Wiener theorem, they have same exponential type. On the other hand, equation (10) implies that the exponential type of these two functions differs by $|\psi(x)|$ so $\psi(x)=0$ for every $x \in \mathbb{R}$.

Now we identify $\varphi$. Let $\Omega$ be the set of all $x$ such that $A(u)(x, y)$ is not identically 0 , thus

$$
\Omega=\left\{x: t \mapsto u\left(t+\frac{x}{2}\right) \overline{u\left(t-\frac{x}{2}\right)} \text { is not } 0 \text { a.e. }\right\}
$$

As $A(u)(x, y)$ is continuous ( $c f$. [3]), we may assume that $\varphi$ is continuous on $\Omega$. As $A(u)(0,0)=$ $A(v)(0,0)=1$, we can assume that $\varphi(0)=0$. Moreover, $\Omega$ is an open set of $\mathbb{R}$, and so a countable union of disjoint intervals. Further, as $A(u)(-x,-y)=\overline{A(u)(x, y)},-\Omega=\Omega$, and we can assume that $\varphi$ is odd. As $A(u)(0,0)=1,0 \in \Omega$, and so we can write $\Omega=\bigcup_{k=-\infty}^{\infty} I_{k}$ with $0 \in I_{0}$ and $I_{-k}=I_{k}$.

Definition Let $f: \mathbb{R} \mapsto \mathbb{C}$ be a measurable function. Then a point $x \in \mathbb{R}$ will be called a Lebesgue point of $f$ if

$$
\frac{1}{|B|} \int_{B} f(y) d y \rightarrow_{B \backslash\{x\}} f(x)
$$

where $\varphi(B) \rightarrow_{B \backslash\{x\}} \alpha$ means that for every $\varepsilon>0$ there exists $r>0$ such that for every ball $B$ of radius $\leq r$ containing $x,|\varphi(B)-\alpha|<\varepsilon$.

Recall that a point $x$ in a set $A \subset \mathbb{R}$ is called a point of density of $A$ if $\frac{|A \cap I|}{|I|} \rightarrow 1$ where $I$ is an interval containing $x$ of length $\eta \rightarrow 0$. In other words a point of density of $A$ is a point of $A$ that is a Lebesgue point of $\chi_{A}$.

Recall that if $f \in L^{1}(\mathbb{R})$ then almost every point of $\mathbb{R}$ is a Lebesgue point of $f$, in particular, almost every point of a measurable set $A$ is a point of density of $A$.

In what follows we assume that every point in the support of $u$ is a Lebesgue point of $u$, and so a Lebesgue point of $|u|$ thus also a point of density of the support of $u$. Let $S=\{x \in \mathbb{R}: u(x) \neq 0\}$ be the support of $u$, and let

$$
E_{x}=\left(S-\frac{x}{2}\right) \cap\left(S+\frac{x}{2}\right)=\left\{t:\left|u\left(t+\frac{x}{2}\right)\right|\left|u\left(t-\frac{x}{2}\right)\right| \neq 0\right\} .
$$


By definition, $\left|E_{x}\right|>0$ if and only if $x \in \Omega$. Note also that, as every point of $E_{x}$ is a point of density of $E_{x}$, if $\left|E_{x}\right|=0$ then $E_{x}=\emptyset$.

Proposition 6 If $S$ is the support of $u$, then $\Omega=S-S$.

Proof. We first prove that $S-S \subset \Omega$. Let $x \notin \Omega$. By definition $\left|E_{x}\right|=0$, so that $E_{x}=\emptyset$, that is, $\left(S-\frac{x}{2}\right) \cap\left(S+\frac{x}{2}\right)=\emptyset$ which yields $x \notin S-S$.

For the reverse inclusion, let $x \in \Omega$, then $\left|E_{x}\right|>0$ which implies that $|(S-x) \cap S|>0$, in particular, there exist $\eta, \xi \in S$ such that $\eta-x=\xi$ thus $x=\eta-\xi \in S-S$.

Lemma 7 If $t_{0}, t_{1}, t_{2}$ are in $S$ then

$$
\varphi\left(t_{2}-t_{1}\right)+\varphi\left(t_{1}-t_{0}\right) \equiv \varphi\left(t_{2}-t_{0}\right) \quad(2 \pi) .
$$

Proof. As $A(u)(x,$.$) and A(v)(x,$.$) are Fourier transforms, we have$

If $x$ and $y$ are in $S$, then

$$
u\left(t+\frac{x}{2}\right) \overline{u\left(t-\frac{x}{2}\right)}=e^{i \varphi(x)} v\left(t+\frac{x}{2}\right) \overline{v\left(t-\frac{x}{2}\right)} .
$$

$$
\frac{1}{(2 \eta)^{2}} \int_{\left|t-\frac{x+y}{2}\right|<\eta} \int_{|s-(x-y)|<\eta} u\left(t+\frac{s}{2}\right) \overline{u\left(t-\frac{s}{2}\right)} d s d t \rightarrow u(x) \overline{u(y)},
$$

as $\eta \rightarrow 0$. If we further assume that $x$ and $y$ are Lebesgue points of $v$, using the continuity of $\varphi$, we obtain

$$
\frac{1}{(2 \eta)^{2}} \int_{\left|t-\frac{x+y}{2}\right|<\eta} \int_{|s-(x-y)|<\eta} e^{i \varphi(s)} v\left(t+\frac{s}{2}\right) \overline{v\left(t-\frac{s}{2}\right)} d s d t \rightarrow e^{i \varphi(x-y)} v(x) \overline{v(y)}
$$

as $\eta \rightarrow 0$ Thus

$$
u(x) \overline{u(y)}=e^{i \varphi(x-y)} v(x) \overline{v(y)} .
$$

Applying relation (12) to $t_{2}, t_{1} \in S$, and to $t_{1}, t_{0} \in S$, we have

$$
u\left(t_{2}\right) \overline{u\left(t_{1}\right)}=e^{i \varphi\left(t_{2}-t_{1}\right)} v\left(t_{2}\right) \overline{v\left(t_{1}\right)} \text { and } u\left(t_{1}\right) \overline{u\left(t_{0}\right)}=e^{i \varphi\left(t_{1}-t_{0}\right)} v\left(t_{1}\right) \overline{v\left(t_{0}\right)}
$$

Multiplying these two relations, we find

$$
u\left(t_{2}\right) \overline{u\left(t_{0}\right)}\left|u\left(t_{1}\right)\right|^{2}=e^{i \varphi\left(t_{2}-t_{1}\right)+i \varphi\left(t_{1}-t_{0}\right)} v\left(t_{2}\right) \overline{v\left(t_{0}\right)}\left|v\left(t_{1}\right)\right|^{2} .
$$

Then, as $|u|=|v|$ almost everywhere, relation (12) leads us to

$$
\varphi\left(t_{2}-t_{1}\right)+\varphi\left(t_{1}-t_{0}\right) \equiv \varphi\left(t_{2}-t_{0}\right)
$$

for almost every $t_{0}, t_{1}, t_{2}$ in $S$, and by continuity of $\varphi$ on $\Omega=S-S$, relation (11) is valid everywhere.

Lemma 8 There exists $\omega \in \mathbb{R}$ and a real sequence $\left(b_{k}\right)_{k \in \mathbb{Z}}$ such that, if there are $t_{0}, t_{1}, t_{2} \in S$ such that $t_{2}-t_{1} \in I_{k}, t_{1}-t_{0} \in I_{k^{\prime}}$ and $t_{2}-t_{0} \in I_{k^{\prime \prime}}$, then

$$
b_{k}+b_{k^{\prime}} \equiv b_{k^{\prime \prime}} \quad(2 \pi)
$$

and, for every $x \in I_{k}, \varphi(x)=\omega x+b_{k}$.

Proof. First, by choosing $t_{0}, t_{1}, t_{2} \in S$ such that $x=t_{2}-t_{1} \in I_{0}, y=t_{1}-t_{0} \in I_{0}$ and $x+y=t_{2}-t_{0} \in I_{0}$, (11) becomes $\varphi(x+y)=\varphi(x)+\varphi(y)$ from which it is easy to see that $\varphi$ is linear, that is, there exists $\omega \in \mathbb{R}$ such that $\varphi(x)=\omega x$ on $I_{0}$.

Now we look at an other connected component $I_{k}$ of $\Omega$. If $t_{0}, t_{1}, t_{2} \in S$ are such that $x=t_{2}-t_{1} \in I_{k}$, $y=t_{1}-t_{0} \in I_{0}$ and $x+y=t_{2}-t_{0} \in I_{k}$, then $\varphi$ satisfies $\varphi(x+y)=\varphi(x)+\omega y$. Thus, for every $k$, there exists $b_{k}$ such that, for $x \in I_{k}, \varphi(x)=\omega x+b_{k}$.

Finally, relation (13) is a direct consequence of relation (11). 
We have proved that there exist $t_{0} \in S, \omega \in \mathbb{R}$, and a sequence $\left(b_{k}\right)$ satisfying (13) such that, for $x \in I_{k}+t_{0}, v(x)=c e^{i \omega x} e^{i b_{k}} u(x)$.

Conversely, assume that the $b_{k}$ 's satisfy condition (9), let $t_{0} \in S$, and define $\varphi(x)=\omega x+b_{k}$ for $x \in I_{k}+t_{0}$. Let $v(x)=\bar{c} e^{i \varphi(x)} u(x)$. Then for $x \in I_{k}+t_{0}$ and almost every $t \in \mathbb{R}$, either there exists $k^{\prime}, k^{\prime \prime}$ such that $t+\frac{x}{2} \in\left(I_{k^{\prime}}+t_{0}\right) \cap S$ and $t-\frac{x}{2} \in\left(I_{k^{\prime \prime}}+t_{0}\right) \cap S,\left(\right.$ as $\left.x=\left(t+\frac{x}{2}\right)-\left(t-\frac{x}{2}\right) \in I_{k}+t_{0}\right)$, $b_{k}=b_{k^{\prime}}-b_{k^{\prime \prime}}$ and

$$
v\left(t-\frac{x}{2}\right) \overline{v\left(t+\frac{x}{2}\right)}=e^{i \omega x} e^{i b_{k}} u\left(t-\frac{x}{2}\right) \overline{u\left(t+\frac{x}{2}\right)}
$$

or $t+\frac{x}{2}$ and $t-\frac{x}{2}$ are not both in $S$ whence

$$
v\left(t-\frac{x}{2}\right) \overline{v\left(t+\frac{x}{2}\right)}=u\left(t-\frac{x}{2}\right) \overline{u\left(t+\frac{x}{2}\right)}=0 .
$$

In particular, $A\left(e^{i \varphi(x)} u\right)(x,)=.e^{i b_{k}+i \omega x} A(u)(x,$.$) , and$

$$
\left|A\left(e^{i \varphi(x)} u\right)(x, .)\right|=|A(u)(x, .)| .
$$

Summarising the previous results, we can now state the following theorem.

Theorem 9 Let $u \in L^{2}(\mathbb{R})$ be a compactly supported function and let $v$ be a restricted ambiguity partner of $u$.

If $\Omega$ is the open set of all $x$ such that $A(u)(x,$.$) is not identically 0$, there exists a locally constant function $\varphi$ on $\Omega$ such that, for every $t_{0}, t_{1}, t_{2}$ belonging to the support of $u$,

$$
\varphi\left(t_{2}-t_{1}\right)+\varphi\left(t_{1}-t_{0}\right) \equiv \varphi\left(t_{2}-t_{0}\right)
$$

and

$$
v(x)=c e^{i \varphi\left(x-a-x_{0}\right)} e^{i \omega x} u(x-a)
$$

for some $a \in \mathbb{R}, \omega \in \mathbb{R}, c \in \mathbb{T}$ and some $x_{0}$ belonging to the support of $u$.

Conversely, every function $v$ of that form is a (restricted) ambiguity partner of $u$.

Remark : Using to the relation $A(\mathcal{F} u)(x, y)=A(u)(-y, x)$ we can easily state this theorem for band limited functions if we replace $u$ and $v$ by $\mathcal{F} u$ and $\mathcal{F} v$ in the above theorem.

Proposition 3 is a particular case of theorem 9 in which condition 14 is reduced to $\varphi$ odd.

In the case $u=\chi_{[a, b]}$ all the zeroes of $A(u)$ are real, thus for every solution $v$ of the ambiguity problem for $u, A(u)$ and $A(v)$ have same zeroes and we have a complete solution of the ambiguity problem for this $u$. Moreover, if every ambiguity partner of $H$ in example 3 is a restricted ambiguity partner of $H$, then example 3 and theorem 9 give every ambiguity partner of $U(t)=a_{0} H(t)+a_{1} H(t-$ 1) $+a_{N} H(t-N)+a_{N+1} H(t-N-1)$.

The theorem essentially states that if $u$ is "simple" (in particular, the support is an interval) then the solutions of the ambiguity problem are "simple", whereas for complicated $u$ (for example, when the support has big gaps) the solutions are also complicated.

3.3. The wide band case. Recall that

$$
W A(u)(a, \tau)=\sqrt{a} \int_{\mathbb{R}} u(t) \overline{u(a(t-\tau))} d t
$$

and that $W A(u)(a, \tau)=\frac{1}{\sqrt{a}} \widetilde{W A}(\mathcal{F} u)(1 / a, \tau / a)$ where

$$
\widetilde{W A}(u)(a, \tau)=\int_{\mathbb{R}} u(t) \overline{u(a t)} e^{2 \pi i \tau t} d t .
$$

We want to know if $|W A(u)|=|W A(v)|$ implies that $v$ is $A$-related to $u$.

We will here briefly review some results for the wide band ambiguity problem for band limited functions or, equivalently, for the $\widetilde{W A}$-ambiguity problem for compactly supported functions. This 
results can be obtained in a similar way to those of the previous section, therefore we will only give sketches of the proofs.

First, let us write $u=u_{0}+u_{1}$ and $v=c_{0} u_{0}+c_{1} u_{1}$ where $u_{0}=\chi_{\left[a_{0}, a_{1}\right]}, u_{1}=\chi_{\left[a_{2}, a_{3}\right]}$. We seek conditions on $a_{0}, a_{1}, a_{2}, a_{3}$ for $\widetilde{W A}\left(u_{0}\right)+\widetilde{W A}\left(u_{1}\right), \widetilde{W A}\left(u_{0}, u_{1}\right), \widetilde{W A}\left(u_{1}, u_{0}\right)$ to have disjoint supports. One then finds functions having wide-band ambiguity functions with same absolute value which are not $A$-related. The precise result is the following :

Proposition 10 Let $\left(a_{k}\right)_{k \geq 0}$ be an increasing sequence of positive real numbers such that

(1) for all $k \geq 0, a_{2(k+1)}>a_{2 k+1} \frac{a_{2 k+1}}{a_{2 k}}$; and

(2) for all $k \geq 0, a_{2(k+1)+1}<a_{2(k+1)} \frac{a_{2(k+1)}}{a_{2 k+1}}$.

Let $\left(u_{k}\right)_{k \geq 0}$ be a sequence of functions in $L^{2}(\mathbb{R})$ such that for all $k \geq 0, \mathcal{F} u_{k}$ is supported in $\left[a_{2 k}, a_{2 k+1}\right]$, and $\sum_{k \geq 0} u_{k} \in L^{2}(\mathbb{R})$.

Finally, let $\left(c_{k}\right)_{k \geq 0}$ be a sequence of complex numbers of modulus 1 . Then, for every $a>0$ and every $\tau \in \mathbb{R}$,

$$
\left|W A\left(\sum_{k \geq 0} c_{k} u_{k}\right)(a, \tau)\right|=\left|W A\left(\sum_{k \geq 0} u_{k}\right)(a, \tau)\right|
$$

whereas, in general $\sum_{k \geq 0} c_{k} u_{k}$ is not $A$-related to $\sum_{k \geq 0} u_{k}$.

We now focus on finding examples of band limited functions for which the solutions of the wide band ambiguity problem for $u$ are all $A$-related to $u$. Again, it is equivalent to work with $\widetilde{W A}$ and compactly supported functions. We will first need the following lemma :

Lemma 11 Let $K$ be a compact set of positive measure such that $0 \notin K$ and let $u \in L^{2}(\mathbb{R})$ be supported in $K$. If $v \in L^{2}(\mathbb{R})$ satisfies

$$
|\widetilde{W A}(v)(a, \tau)|=|\widetilde{W A}(u)(a, \tau)|
$$

for all $a>0, \tau \in \mathbb{R}$, then $v$ is also compactly supported.

Proof. $K$ being a compact not containing 0 , we can find an $\varepsilon>0$ and an $\eta>0$ such that if $|x|<\varepsilon$ or $|x|>\eta$ then $u(x)=0$. But then,

$$
\widetilde{W A}(u)(a, \tau)=\int_{\mathbb{R}} u(t) \overline{u(a t)} e^{2 \pi i \tau t} d t=0
$$

if $a>\frac{\eta}{\varepsilon}$ or if $a<\frac{\varepsilon}{\eta}$, and so $v(t) \overline{v(a t)}=0$ a.e. Thus, if $t_{0}$ is any Lebesgue point of $v$ that is in the support of $v, v(t)=0$ if $|t|>\frac{\eta}{\varepsilon}\left|t_{0}\right|$ and if $|t|<\frac{\varepsilon}{\eta}\left|t_{0}\right|$. Hence $v$ is compactly supported.

Remark : The Paley-Wiener theorem implies that if $u$ is supported in $[-\eta, \eta]$, so is $v$ and the previous proof tells us that if $K$ is contained in $[-\eta,-\varepsilon] \cup[\varepsilon, \eta]$, so is the support of $v$.

To go further, we again use the methods of section 3. A second appeal to the Paley Wiener theorem tells us that $\widetilde{W A}(u)(a, \tau)$ and $\widetilde{W A}(v)(a, \tau)$ are entire functions of order 1 in $\tau$.

Here again, $\widetilde{W A}(u)$ and $\widetilde{W A}(v)$ need not have the same zeroes. For instance, let $N \geq 4$ be an integer, and let

$$
u=a_{0} \chi_{[1,2]}+a_{1} \chi_{[4,8]}+a_{N} \chi_{\left[2^{2 N}, 2^{2 N+1}\right]}+a_{N+1} \chi_{\left[2^{2 N+2}, 2^{2 N+3}\right]}
$$

and

$$
v=b_{0} \chi_{[1,2]}+b_{1} \chi_{[4,8]}+b_{N} \chi_{\left[2^{2 N}, 2^{2 N+1}\right]}+b_{N+1} \chi_{\left[2^{2 N+2}, 2^{2 N+3}\right]}
$$

Then if $\left|a_{i}\right|=\left|b_{i}\right|$ for $i=0,1, N, N+1$ and if

$$
\arg b_{0}-\arg b_{1}-\arg b_{N}+\arg b_{N+1} \equiv \arg a_{0}-\arg a_{1}-\arg a_{N}+\arg a_{N+1}
$$


but $\arg b_{0}-\arg b_{1} \not \equiv \arg a_{0}-\arg a_{1}(2 \pi)$ and $\arg b_{N}-\arg b_{N+1} \not \equiv \arg a_{N}-\arg a_{N+1}(2 \pi) \operatorname{then} \widetilde{W A}(u)$ and $\widetilde{W A}(v)$ have same modulus, but not same zeroes, neither do $\widetilde{W A}(u)$ and $\widetilde{W A}(C Z v)$ have same zeroes.

In what follows, we assume that $\widetilde{W A}(u)$ and $\widetilde{W A}(v)$ have same zeroes and solve the following restricted wide band ambiguity problem :

Problem (Restricted Wide Band Ambiguity) Given a compactly supported function $u \in L^{2}(\mathbb{R})$, what is the set of all $v \in L^{2}(\mathbb{R})$ such that

$$
|\widetilde{W A}(u)(a, \tau)|=|\widetilde{W A}(v)(a, \tau)|
$$

for all $a>0, \tau \in \mathbb{R}$, and such that $\widetilde{W A}(u)$ and $\widetilde{W A}(v)$ have same zeroes ?

Using Hadamard's factorisation theorem we can write

$$
\widetilde{W A}(v)(a, \tau)=e^{i(\varphi(a)+\psi(a) \tau) \widetilde{W A}(u)(a, \tau)}
$$

with $\varphi, \psi: \mathbb{R} \mapsto \mathbb{R}$, from which we deduce again that $\psi=0$, as in the narrow band case.

We will now identify $\varphi$. Let $\Omega$ be the set of all $a$ such that $\widetilde{W A}(u)(a, \tau)$ is not identically 0 , thus :

$$
\Omega=\{a: t \mapsto u(t) \overline{u(a t)} \text { is not } 0 \text { a.e. }\}
$$

As $\widetilde{W A}(u)(a, \tau)$ is a continuous function of $(a, \tau), \Omega$ is an open set of $\mathbb{R}$ and so a countable union of disjoint intervals, $\Omega=\bigcup_{k \in \mathbb{N}} I_{k}$.

Now, as

$$
\widetilde{W A}(u)(a, \tau)=e^{i \varphi(a)} \widetilde{W A}(v)(a, \tau),
$$

as $\widetilde{W A}(u)(a, \tau)$ and $\widetilde{W A}(v)(a, \tau)$ are continuous, and as

$$
\widetilde{W A}(u)(1,0)=\widetilde{W A}(v)(1,0)=1,
$$

we may assume that $\varphi$ is continuous and that $\varphi(1)=0$, in particular $|u|=|v|$ almost everywhere.

We again assume that every point in the support $S$ of $u$ is a Lebesgue point of $u$, and so a Lebesgue point of $|u|$ and a point of density of $S$. We also assume that $0 \notin S$.

The following proposition can be obtained in a similar way to proposition 6 :

Proposition 12 Let $u \in L^{2}(\mathbb{R})$ be supported in a compact set not containing 0 . Let $S_{+}$be the set of positive density points of the support of $u$ and $S_{-}$the set of negative density points of the support of $u$. Then

$$
\Omega=\frac{S_{+}}{S_{+}} \cup \frac{S_{-}}{S_{-}}=\left\{\frac{x}{y}: x, y \in S_{+}\right\} \cup\left\{\frac{x}{y}: x, y \in S_{-}\right\} .
$$

But now, $\widetilde{W A}$ is the Fourier transform of $u(t) \overline{u(a t)}$ in the $\tau$ variable and

so that

$$
\widetilde{W A}(u)(a, \tau)=e^{i \varphi(a)} \widetilde{W A}(v)(a, \tau)
$$

Thus, if $t_{1}, t_{2} \in S$, then

$$
u(t) \overline{u(a t)}=e^{i \varphi(a)} v(t) \overline{v(a t)} .
$$

$$
u\left(t_{1}\right) \overline{u\left(t_{2}\right)}=e^{i \varphi\left(\frac{t_{2}}{t_{1}}\right)} v\left(t_{1}\right) \overline{v\left(t_{2}\right)}
$$

and, as for lemma 7, we obtain

Lemma 13 If $t_{0}, t_{1}, t_{2}$ are in $S$, then

$$
\varphi\left(\frac{t_{0}}{t_{1}}\right)+\varphi\left(\frac{t_{1}}{t_{2}}\right) \equiv \varphi\left(\frac{t_{0}}{t_{2}}\right)
$$


From this we deduce the following analogue of lemma 8 :

Lemma 14 There exist $\lambda \in \mathbb{R}$ and a real sequence $\left(b_{k}\right)_{k \in \mathbb{Z}}$ such that, if there are $t_{0}, t_{1}, t_{2} \in S$ such that $\frac{t_{0}}{t_{1}} \in I_{k}, \frac{t_{1}}{t_{2}} \in I_{k^{\prime}}$ and $\frac{t_{0}}{t_{2}} \in I_{k^{\prime \prime}}$, then

$$
b_{k}+b_{k^{\prime}} \equiv b_{k^{\prime \prime}}
$$

and, for every $k \in \mathbb{N}, x \in I_{k}, \varphi(x)=\lambda \log x+b_{k}$.

Translating the results from $\widetilde{W A}$ to $W A$ we finally have the following analogue of theorem 9 :

Theorem 15 Let $K$ be a compact set not containing 0 , and let $u \in L^{2}(\mathbb{R})$ whose spectrum is in $K$. Let $v$ be such that $\mathcal{F} v$ is a solution of the restricted wide band ambiguity problem for $\mathcal{F} u$. If $\Omega$ is the open set of all $x$ such that $\widetilde{W A}(\mathcal{F} u)(x,$.$) is not identically 0$, there exists a locally constant function $\varphi$ on $\Omega$ such that for every $t_{0}, t_{1}, t_{2}$ belonging to the spectrum of $u$

$$
\varphi\left(\frac{t_{0}}{t_{1}}\right)+\varphi\left(\frac{t_{1}}{t_{2}}\right) \equiv \varphi\left(\frac{t_{0}}{t_{2}}\right)
$$

and

$$
\mathcal{F} v(x)=c e^{i \varphi\left(\frac{x}{t_{0}}\right)} e^{i \lambda \log x} \mathcal{F} u(x)
$$

for some $c \in \mathbb{T}, \lambda \in \mathbb{R}$ and some $t_{0}$ belonging to the spectrum of $u$.

Conversely, every function $v$ of that form is such that $\mathcal{F} v$ is a solution of the restricted wide band ambiguity problem for $\mathcal{F} u$.

\section{Conclusion}

The radar ambiguity problem is far from being solved. The phenomenon of zero flipping is certainly not understood and indeed, in some cases the extra structure of ambiguity functions (cf. [3]) may well intervene to render it superfluous. That is, no zero-flipping may be allowed.

This article improves our understanding of the radar ambiguity problem in three ways:

(1) it completely solves the radar ambiguity problem up to zero flipping;

(2) it provides new examples where the solution set is minimal (for example, when $u=\chi_{[a, b]}$ ) or near to minimal, that is, without zero flipping (for instance, when $u$ is a sum of translates of $\chi_{[a, b]}$, via Proposition 2);

(3) it provides a method of obtaining many functions $u$ for which the solution set is much bigger then expected, in particular, it contains functions that are far from being Heisenberg related to $u$ (example 3 ).

It would be particularly interesting to explain what happens for functions of the form $P(x) e^{-x^{2}}$ with $P$ a polynomial. In this case $A(u)$ is essentially a polynomial and the additional structure alluded to above comes into play, forbidding zero flipping. We believe that this result extends to functions of the form $P(x) e^{-x^{2}}$ with $P$ an entire function of order less than 2. (One can easily show that, if $u$ is of the form $P(x) \exp \left(-x^{2}\right)$ with $P$ an entire function of order $\alpha<2$ and if $v$ is an ambiguity partner of $u$, then $v$ is of the form $M(\omega) S(a)\left(Q(x) \exp \left(-x^{2}\right)\right.$ ) with $Q$ an entire function of same order $\alpha$.)

Such a result would be of theoretical significance. Recall that Bueckner associated to every function $u$ a Hilbert-Schmidt operator $K_{u}$. It turns out that $v$ solves the radar ambiguity problem for $u$ if and only if $K_{u}$ and $K_{v}$ are "similar". A careful reading of [5] also shows that if $K_{u}$ is of finite rank, then $u$ is of the form $P(x) e^{-x^{2}}$ with $P$ an entire function of order at most 2 . As finite rank operators approximate Hilbert-Schmidt operators, we think that the result for finite rank operators and some considerations on the "speed" of approximation should also bring new results.

\section{THANKS}

The author wishes to thank A. Bonami and W. Moran for valuable conversations and the CSSIP branch at Flinders University for partial financial support. 


\section{NOTE ADDED IN PROOF}

After the first submission of this paper the author became aware of certain earlier examples of nontrivial Pauli partners and ambiguity partners. In [13], A.E.J.M. Janssen gave other examples of Pauli partners and found non-trivial ambiguity-partners of Proposition 3 with a method based on the Zak transform $\mathcal{Z} f(\tau, \omega)=\sum_{k \in \mathbb{Z}} e^{-2 i \pi k \omega} f(\tau+k)$. His example 1 can also lead to another proof of Theorem 2 : let $\left(A_{k}\right)_{k \in \mathbb{N}},\left(B_{k}\right)_{k \in \mathbb{N}}$ be two partitions of $[-1 / 2,1 / 2]$ into open sets and let $\varphi_{k} \in L^{2}([-1 / 2,1 / 2])$ be supported in $A_{k} \times B_{k}$. Let $f_{k}=\mathcal{Z}^{-1} \varphi_{k}$, then $f=\sum_{k \in \mathbb{N}} f_{k}$ and $g=\sum_{k \in \mathbb{N}} c_{k} f_{k}$ with $\left|c_{k}\right|=1$ are non-trivial Pauli partners that are non-trivial solutions of the phase-retrieval problem, however these examples are essentially different from those in Theorem 2 . Note also that $f$ and $\hat{f}$ are never compactly supported, nevertheless taking a smooth $\varphi_{k}$ leads to $f \in \mathcal{S}(\mathbb{R})$.

\section{REFERENCES}

[1] Altes R.A. Some invariance properties of the wide-band ambiguity function. Jour. of the Acoustical Soc. Amer., 53:1154-1160, 1973.

[2] Auslander L. and Gretner I. Wide-band ambiguity functions and the $a x+b$ group. In Signal processing part I: Signal Processing Theory, volume 22 of I.M.A. vol in Math and its Appl, pages 1-12, 1990.

[3] Auslander L. and Tolimieri R. Radar ambiguity functions and group theory. SiAM J. Math Anal, 16:577-6, 1985.

[4] Barakat R. and Newsam G. Necessary conditions for a unique solution to two dimensional phase recovery. $J$. Math. Phys, 25:3190-3193, 1984.

[5] Bueckner H.F. Signals having the same ambiguity functions. Technical Report 67-C-456, General Electric, Research and Development Center, Schnectady, N.Y., 1967.

[6] Corbett J.V. and Hurst C.A. What is needed to determine a state. manuscript.

[7] Corbett J.V. and Hurst C.A. Are wave functions uniquely determined by their position and momentum distributions? J. Austral. Math. Soc B, 20:182-201, 1978.

[8] DE BUdA R. Signals that can be calculated from their ambiguity function. IEEE Trans. Information Theory, IT16:195-202, 1970.

[9] Friedman C. N. Some remarks on Pauli data in quantum mechanics. J. Austral. Math. Soc. Ser. B, 30:298-303, 1989.

[10] Grünbaum F.A. A remark on the radar ambiguity function. IEEE Trans. Information Theory, 30:126-127, 1984.

[11] Hurt N.E. Phase Retrieval and Zero Crossing (Mathematical Methods in Image Reconstruction). Math. and Its Appl. Kluwer Academic Publisher, 1989.

[12] Ismagilov R.S. On the Pauli problem. Funksional Anal i Prilozhen, 30:82-84, 1996. in russian, translation in Funct. Anal. Appl 30 (1996) 138-140.

[13] Janssen A.J.E.M. The Zak transform and some counterexamples in time-frequency analysis. IEEE Trans. Information Theory, 38:168-171, 1992.

[14] Katznelson Y. An Introduction to Harmonic Analysis. Dover, 1976.

[15] Klibanov M. V., Sacks P.E. and Tikhonravov A.V. The phase retrieval problem. Inverse problems, 11:1-28, 1995.

[16] Pauli W. Die allgemeinen Prinzipien der Wellenmechanik. Handbuch der Physik, V:17, 1932.

[17] Reichenbach H. Philosophic Foundations of Quantum Mechanics. Univesity of California Press, Berkeley, 1944.

[18] Rosenblatt J. Phase retrieval. Comm. Math. Phys., 95:317-343, 1984.

[19] Stefanescu I.S. On the phase retrieval problem in two dimensions. J. Math. Phys, 26:2141-2160, 1985.

[20] Swick D.A. An ambiguity function independent of assumption about bandwidth and carrier frequency. Technical report, NRL Repport, 15 DEC 1966.

[21] Titchmarsh E. The Theory of Functions. Oxford University press, 1939. 2nd ed.

[22] Vogt A. Position and momentum distributions do not determine the quantum mechanical state. In A.R. Marlow, editor, Mathematical Foundations of Quantum Theory. Academic Press, 1978. 365-372.

[23] Walter A. The question of phase retrieval in optics. Opt. Acta, 10:41-49, 1963.

[24] WILcox C.H. The synthesis problem for radar ambiguity functions. Technical Report 157, MRC Tech. Summary Report, 1960. republished in Radar and Sonar part I vol 32 I.M.A. vol in Math. and its Appl,1991, 229-260.

[25] Woodward P.M. Probability and Information Theory with Applications to RADAR. Pergamon, 1953.

Université d'Orléans, Faculté des Sciences, Département de Mathématiques, BP 6759, F 45067 ORLEANS CEDEX 2, FRANCE

E-mail address: jaming@labomath.univ-orleans.fr 